\title{
Dissecting the basis of novel trait evolution in a radiation with widespread phylogenetic discordance
}

Meng Wu ${ }^{1}$, Jamie L. Kostyun ${ }^{1,2}$, Matthew W. Hahn ${ }^{1,3}$, and Leonie C. Moyle ${ }^{1, *}$

${ }^{1}$ Department of Biology, Indiana University, Bloomington, Indiana, U.S.A. 47405

${ }^{2}$ Department of Plant Biology, University of Vermont, Burlington, Vermont, U.S.A., 05401

${ }^{3}$ Department of Computer Science, Indiana University, Bloomington, Indiana, U.S.A.

1247405

*Corresponding author: Email: $\underline{\text { lmoyle@indiana.edu }}$

RRH: Trait evolution under widespread discordance 


\section{ABSTRACT}

Phylogenetic analyses of trait evolution can provide insight into the evolutionary

25 processes that initiate and drive phenotypic diversification. However, recent

26 phylogenomic studies have revealed extensive gene tree-species tree discordance, which

27 can lead to incorrect inferences of trait evolution if only a single species tree is used for

28 analysis. This phenomenon-dubbed "hemiplasy"-is particularly important to consider

29 during analyses of character evolution in rapidly radiating groups, where discordance is

30 widespread. Here we generate whole-transcriptome data for a phylogenetic analysis of 14

31 species in the plant genus Jaltomata (the sister clade to Solanum), which has experienced

32 rapid, recent trait evolution, including in fruit and nectar color, and flower size and shape.

33 Consistent with other radiations, we find evidence for rampant gene tree discordance due

34 to incomplete lineage sorting (ILS) and several introgression events among the well-

35 supported subclades. Since both ILS and introgression increase the probability of

36 hemiplasy, we perform several analyses that take discordance into account while

37 identifying genes that might contribute to phenotypic evolution. Despite discordance, the

38 history of fruit color evolution in Jaltomata can be inferred with high confidence, and we

39 find evidence of de novo adaptive evolution at individual genes associated with fruit

40 color variation. In contrast, hemiplasy appears to strongly affect inferences about floral

41 character transitions in Jaltomata, and we identify candidate loci that could arise either

42 from multiple lineage-specific substitutions or standing ancestral polymorphisms. Our

43 analysis provides a generalizable example of how to manage discordance when

44 identifying loci associated with trait evolution in a radiating lineage. 
bioRxiv preprint doi: https://doi org/10.1101/201376 : this version posted October 11 2017. The copyright holder for this preprint (which was not certified by peer review) is the author/funder, who has granted bioRxiv a license to display the preprint in perpetuity. It is made available under aCC-BY-NC 4.0 International license.

46 Key words: phylogenomics, rapid radiation, hemiplasy, convergence, Jaltomata, Solanum 


\section{INTRODUCTION}

Phylogenies contribute to our understanding of the evolutionary history of traits

50 (Felsenstein, 1985). When the patterns of relationship among species is known, robust

51 inferences about character state evolution can be made, including the number of times a

52 character evolved, the direction of character evolution, and the most likely ancestral

53 character state. Phylogenies can also reveal whether lineages with similar phenotypic

54 traits have evolved these via independent evolution (convergence or parallelism) or

55 whether a single origin is more likely (Wake et al., 2011). The recent use of whole

56 genomes or transcriptomes to make phylogenetic inferences from thousands to millions

57 of sites ("phylogenomics"), has succeeded in its aim of generating species trees with high

58 levels of statistical support. However, other genome-wide analyses have begun to reveal

59 unexpected complexities in the evolutionary history of rapidly radiating lineages-

60 including widespread gene tree discordance due to incomplete lineage sorting and/or

61 introgression (Degnan \& Rosenberg, 2009). This frequent discordance among individual

62 gene trees can amplify incorrect inferences of trait evolution on even well-supported

63 species trees. In particular, when a trait is determined by genes whose topologies do not

64 match the species topology, incorrect inferences of homoplasy (independent evolution of

65 the same character state) are substantially elevated - a phenomenon known as

66 'hemiplasy' (Avise \& Robinson, 2008; Hahn \& Nakhleh, 2016; Storz, 2016). Because

67 understanding trait evolution-including the underlying genetic changes—is of particular

68 interest in species radiations, extra care must be taken to consider and account for the

69 influence of hemiplasy in these cases. 
The fraction of the genome affected by hemiplasy will depend upon the amount

71 and sources of gene tree discordance in a clade. In rapidly radiating species groups,

72 widespread discordance has been attributed to the effects of both incomplete lineage

73 sorting (ILS) and introgression between lineages (Degnan \& Rosenberg, 2009). ILS

74 affects gene tree topologies when segregating ancestral variation is maintained through

75 consecutive speciation events (Maddison, 1997). Because the effect of ILS is proportional

76 to ancestral population size, and inversely proportional to the time between speciation

77 events (Pamilo \& Nei, 1988), ILS is expected to be particularly exaggerated in radiations

78 where a diverse ancestral population undergoes rapid speciation. Indeed, gene tree

79 discordance has been noted for a substantial fraction of the genome in rapidly radiating

80 groups, including the Drosophila simulans sub-clade (Garrigan et al., 2012), African

81 cichlid fishes (Brawand et al., 2014), wild tomatoes (Pease et al., 2016), and the genus

82 Arabidopsis (Novikova et al., 2016). When there is introgression, discordance emerges

83 because genes that are introgressed among lineages will show historical patterns of

84 relatedness that differ from the loci in the genome into which they are introduced.

85 Substantial introgression has also been identified among rapidly radiating lineages

86 through genome-wide analysis, including in Xiphophorus fishes (Cui et al., 2013),

87 Heliconius butterflies (Martin et al., 2013), Darwin’s finches (Lamichhaney et al., 2015),

88 and Anopheles mosquitoes (Fontaine et al., 2015).

89 Both ILS and introgression contribute to hemiplasy because they cause a

90 proportion of gene trees to disagree with the species tree (Avise \& Robinson, 2008; Hahn

91 \& Nakhleh, 2016; Storz, 2016). Specifically, the probability of hemiplasy is expected to

92 be 1) proportional to the fraction of gene trees that are discordant with the species tree; 
93 and 2) negatively correlated with the branch length leading to clades with similar

94 phenotypes (Hahn \& Nakhleh, 2016). A higher proportion of discordant gene trees

95 increases the probability that a character of interest is underpinned by genes that have a

96 tree topology that differs from the species tree; shorter branch lengths increase the chance

97 of incorrectly inferring homoplasy, as they leave relatively little time for convergent

98 evolution to happen (Hahn \& Nakhleh, 2016). Both conditions are expected to be

99 exaggerated specifically in rapidly diversifying groups. Therefore, in these cases mapping

100 characters onto a single species tree has a substantially elevated risk of incorrectly

101 inferring the number of times a trait has evolved and the timing of trait changes (Avise \&

102 Robinson, 2008; Hahn \& Nakhleh, 2016; Storz, 2016). Hemiplasy also affects inferences

103 about the specific loci inferred to underlie trait transitions because, when ILS or

104 introgression are common, the substitutions underlying trait transitions may occur on

105 gene trees that are discordant with the species tree (Mendes et al., 2016). Accordingly,

106 genome-wide analyses must take into account the extent and distribution of ILS and

107 introgression if they are to accurately infer the number and timing of evolutionary

108 changes in specific traits, and the genes underlying these changes.

109 In this study, we used genome-wide data to investigate the morphologically and

110 ecologically diverse plant genus Jaltomata, in which several key trait transitions appear

111 to have occurred in parallel (Miller et al., 2011), and have been inferred to be

112 independent convergent responses to similar selective pressures. However, because trait

113 diversification has occurred in a relatively short period in this group, the probability of

114 hemiplasy is also expected to be elevated. Our main goals were to assess the timing of

115 lineage and trait diversification in the group, and to identify sources of genetic variation 
116 that potentially contribute to rapid trait diversification in Jaltomata, while taking into

117 account the potential for hemiplasy. To do so, we generated a clade-wide whole-

118 transcriptome dataset and explicitly evaluated alternative scenarios to explain trait

119 evolution by: 1) reconstructing phylogenetic relationships among target species, and

120 evaluating the extent of discordance with the resulting inferred species tree; 2) evaluating

121 patterns of trait variation and evolution in key reproductive (flower and fruit) characters,

122 in the context of best and least supported nodes in this tree; and, 3) evaluating specific

123 scenarios of the genetic changes associated with this trait evolution, in order to identify

124 candidate loci that might be causally responsible. Our results imply two different

125 scenarios of trait evolution for fruit color versus floral traits, reflecting the different

126 amounts of hemiplasy associated with the two traits. While fruit color evolution in

127 Jaltomata could be confidently inferred-along with potential de novo molecular changes

128 on the relevant evolutionary branches - inferring the history of floral trait evolution and

129 the potential contributing loci requires more careful treatment that considers the high

130 probability of hemiplasy.

\section{MATERIALS AND METHODS}

\section{$133 \quad$ Study system}

The plant genus Jaltomata includes approximately 60-80 species, distributed from

135 the southwestern United States through to the Andes of South America (Mione, 1992;

136 Mione et al., 2015) (Figure 1). It is the sister genus to Solanum, the largest and most

137 economically important genus in the family Solanaceae (Olmstead et al., 2008; Särkinen

138 et al., 2013). Species of Jaltomata live in a wide range of habitats, and are phenotypically 
139 diverse in vegetative, floral, and other reproductive traits (Mione 1992; Kostyun \& Moyle

140 2017). Floral diversity is particularly pronounced in Jaltomata. In comparison to closely

141 related clades (including Solanum, Capsicum, and Lycianthes) which predominately have

142 'flattened' rotate corollas (petals) (Knapp, 2010), Jaltomata species exhibit a variety of

143 corolla shapes, including rotate, as well as campanulate and tubular (Miller et al., 2011).

144 All Jaltomata species also produce at least some nectar, including noticeably red- or

145 orange-colored nectar in some lineages, while nectar is not produced by species in

146 Solanum.

Species also differ in fruit color, and fruit color variation appears to characterize

148 major subgroups within the genus as separate dark purple, red, and orange-fruited clades

149 (Miller et al., 2011; Särkinen et al., 2013). Several species also have green fruit at

150 maturity, although these lineages appear to be distributed across the three major

151 Jaltomata clades, suggesting multiple convergent losses of fruit pigment (Miller et al.,

152 2011). The first molecular phylogeny of this genus (Miller et al., 2011) was inferred from

153 a single gene (waxy), and indicates that the lineage of species with red fruits is sister to

154 the rest of the genus. However, a more recent study using seven loci (5 plastid and 2

155 nuclear, including waxy) showed a conflicting topology, with purple-fruited lineages

156 sister to the remaining groups, and red-fruited lineages more closely related to lineages

157 with orange fruits (Särkinen et al., 2013). The inconsistency between the two studies

158 might be the result of using few loci, or of reconstructions performed with loci that have

159 different evolutionary histories. 

previously identified major clades (Miller et al., 2011), and that span representative floral

164 diversity within the genus (Figure 2A, Table S1). Tissues for RNA extraction included

165 seven reproductive tissues (ranging from early bud, to mature pollinated flower, to early

166 fruit) and four vegetative tissues (roots, early leaf buds, and young and mature leaves),

167 from a single representative individual of each target species (see Supplementary text).

168 All sampled individuals were housed at the Indiana University research greenhouse,

169 under standardized temperature $\left(15-20^{\circ} \mathrm{C}\right)$, watering (twice daily), and lighting (16-hour 170 days) conditions.

172 was collected into pre-chilled tubes under liquid nitrogen, each sample was individually 173 ground under liquid nitrogen, and RNA was extracted from $<100 \mathrm{mg}$ ground tissue using

174 the Qiagen Plant RNeasy kit. RNA quality/quantity was checked via Nanodrop (Thermo

175 Fisher Scientific); qualified samples of >50ng/uL with 260/280 and 260/230 between 1.8-

1762.0 were brought to the IU Center for Genomics and Bioinformatics (CGB) for library

177 preparation. Separate reproductive and vegetative libraries for RNA-seq were prepared

178 by pooling equi-molar RNA samples from all reproductive tissues, and all vegetative

179 tissues, respectively, for each species. Both reproductive and vegetative libraries were

180 prepared for all species except for J. grandibaccata, for which only vegetative RNA

181 could be obtained.

Libraries were sequenced using 100-bp paired-end reads in a single lane of 
185 quality reads and ambiguous bases, and trimming adapter ends (see Supplementary text).

186 The retained reads (length $>50 \mathrm{bps}$ ) from vegetative and reproductive transcriptomes of

187 the same species were combined prior to assembly using Trinity with the default settings

188 (Grabherr et al., 2011). The open reading frame of each assembled transcript was

189 predicted using TransDecoder v.2.0.1 with default settings (Haas et al., 2013). All the

190 predicted protein-coding sequences within each Jaltomata species transcriptome were

191 reduced using CD-HIT v4.6 with -c 0.99 -n 10 (Fu et al., 2012). Each sequence in the

192 assembled transcriptome was presented as a haploid representative of particular transcript.

193 To include domestic tomato (Solanum lycopersicum) as the outgroup in the following

194 analyses, we also downloaded the annotated tomato protein-coding sequences from

195 SolGenomics (ftp://ftp.solgenomic.net).

197 Protein-coding gene ortholog identification

198 To infer orthologous gene clusters, we followed a pipeline designed for

199 transcriptome data in non-model species, that begins with an all-by-all BLAST search

200 followed by several steps that iteratively split sub-clusters of homologs at long internal

201 branches, until the subtree with the highest number of non-repeating/non-redundant taxa

202 is obtained (Yang et al., 2015; Yang \& Smith, 2014) (see Supplementary text, Figure S1).

203 For the primary analyses, our homologous clusters were required to include a $S$.

204 lycopersicum (tomato) homolog in each cluster. For one of our downstream analyses

205 (molecular evolution on the basal branch leading to Jaltomata; see below) we also used

206 Capsicum annuum (pepper) sequence data. To do so, we added the $C$. annuum sequences

207 if the tomato sequence in the orthologous cluster had an identified 1-to-1 ortholog in a 
208

209

210

211

212

213

214

215

216

217

218

219

220

221

222

223

224

225

226

227

228

229

230

pepper gene model (http://peppersequence.genomics.cn).

We prepared multiple sequence alignments of orthologous genes using the program GUIDANCE v.2.0 (Sela et al., 2015) with PRANK v.150803 (Löytynoja \& Goldman, 2005) as the alignment algorithm, with codons enforced and ten bootstrap replicates. As a final quality check, we further removed poorly aligned regions using a sliding window approach that masked any 15-bp window from alignment if it had more than three mismatches (indels/gaps were not counted) between ingroup sequences, or had more than five/seven mismatches when tomato/pepper sequences were included. After this process, any alignment with more than $20 \%$ of its sequence masked was removed from the analysis. The resulting sequence alignments were converted to the Multisample Variant Format (MVF), and then genetic distances were computed in all possible pairs of species using the program MVFtools (Pease \& Rosenzweig, 2015).

\section{Estimating the amount of shared variation}

To quantify the amount of variation shared among species and subclades in Jaltomata, the reads from all 14 species were mapped to the reference tomato genome (The Tomato Genome Consortium, 2012) using STAR v2.5.2 (Dobin et al., 2013). SAM files generated were converted to sorted BAM files using SAMtools v. 0.1.19 (Li et al., 2009). SAMtools mpileup was then used to call alleles from the BAM files for all lineages. VCF files were processed into MVF files using $v c f 2 m v f$ from the MVFtools package (Pease \& Rosenzweig, 2015), requiring non-reference allele calls to have Phred scores $\geq 30$ and mapped read coverage $\geq 10$. Based on the MVF files, the numbers of variant sites shared between different subclades of Jaltomata species were counted. 


\section{$232 \quad$ Phylogenetic analysis}

233 We used four different, but complementary, inference approaches to perform

234 phylogenetic reconstruction: 1) maximum likelihood applied to concatenated alignments;

235 2) consensus of gene trees; 3) quartet-based gene tree reconciliation; and 4) Bayesian

236 concordance of gene trees. Because these four approaches use different methods to

237 generate a phylogeny, we applied all four to evaluate the extent to which they generated

238 phylogenies that disagreed, as well as to identify the specific nodes and branches that

239 were robust to all methods of phylogenetic reconstruction. For the concatenation

240 approach, we first aligned all orthologous genes $(n=6431)$, and then used those

241 alignments to build a supermatrix of sequences $(6,223,350$ sites in total). The species tree

242 was then inferred by maximum likelihood using the GTRCAT model in RAxML v8.23

243 with 100 bootstraps (Stamatakis, 2006). We also inferred chromosome-concatenated

244 phylogenies with this method. The other three methods (i.e. consensus, quartet-based, and

245 Bayesian concordance) infer species relationships based on gene trees. First, we inferred

246 the majority rule consensus tree with internode certainty (IC) and internode certainty all

247 (ICA) support scores using RAxML with the option for Majority Rule Extended

248 (Salichos \& Rokas, 2013). Second, we inferred a quartet-based estimation of the species

249 tree by using the program ASTRAL v.4.10.9 with 100 bootstraps (Mirarab \& Warnow,

250 2015). All 6431 RAxML gene trees were used as input in the consensus and quartet-

251 based approaches. Finally, the Bayesian primary concordance tree and associated

252 concordance factors (CFs; indicative of the posterior probability of gene trees supporting

253 a node) at each internode of the primary concordance tree was computed in the program 
254 BUCKy v1.4.4 (Larget et al., 2010). Because BUCKy is computationally intensive for a

255 large number of input gene trees, we only used orthologous gene sets that were

256 potentially informative for resolving gene trees in these analyses. Specifically, we utilized

2571517 genes that showed average bootstrap values $>50$ across the RAxML-inferred gene

258 trees. (These are generally the loci with sufficient genetic variation across the tree to

259 provide information about branch support.) The input of a posterior distribution of gene

260 trees was generated from an analysis with MrBayes v3.2 (Huelsenbeck \& Ronquist,

261 2001). We ran MrBayes for one million Markov chain Monte Carlo (MCMC) generations,

262 and every 1000th tree was sampled. After discarding the first half of the 1000 resulting

263 trees from MrBayes as burnin, BUCKy was performed for one million generations with

264 the default prior probability that two randomly sampled gene trees share the same tree

265 topology is $50 \%(\alpha=1)$ (Larget et al., 2010).

266 All inferred species trees were plotted using the R package "phytools" (Revell, 2012).

267 To estimate dates of divergence, we used the function "chronos" in the R package "ape"

268 (Paradis et al., 2004) to fit a chronogram to the RAxML genome-wide concatenated

269 phylogeny by using penalized likelihood and maximum likelihood methods implemented

270 in chronos. Times were calibrated using a previous estimate of the divergence time

271 between Solanum and Jaltomata at 17 Ma (Särkinen et al., 2013). To visualize gene tree

272 discordance, a "cloudogram" of 183 gene trees with average node bootstrap values

273 greater than 70 was prepared using DensiTree v 2.2.1 (Bouckaert, 2010).

276 The number of sampled species (14) is small compared to the size of this clade (60-

27780 species), and sparse taxon sampling is known to affect the reconstruction of ancestral 
278 character states (Heath et al., 2008). Nonetheless, to assess whether our confidence in the

279 number and placement of transitions generally differs among different traits in our clade,

280 we reconstructed ancestral states for fruit color, nectar color, nectar volume and corolla

281 shape. We used the ultrametric species tree inferred from the RAxML genome-wide

282 concatenated phylogeny and the distribution of traits at the tips of phylogeny (Figure 2A)

283 as input. While nectar volume is a quantitative trait, the other three traits are categorical

284 (fruit color: purple/red/orange/green; nectar color: red/clear; corolla shape:

285 rotate/campanulate/tubular). Ancestral character states were inferred using the standard

286 maximum likelihood method with the equal rates model "ER" within the phytools

287 package (Revell, 2012), which models the evolution of discrete-valued traits using a

288 Markov chain, and the evolution of continuous-valued traits using Brownian motion.

\section{$\underline{\text { Testing for introgression }}$}

We searched for evidence of post-speciation gene flow, or introgression, using the

292 ABBA-BABA test (Durand et al., 2011; Green et al., 2010) on the concatenated

293 orthologous sequence alignment ( $\mathrm{n}=6431$ and $6,223,350$ sites in total). The ABBA-

294 BABA test detects introgression by comparing the frequency of alternate ancestral ("A")

295 and derived ("B") allele patterns among four taxa. In the absence of gene flow, the

296 alternate patterns ABBA and BABA should be approximately equally frequent, given the

297 equal chance of either coalescence pattern under incomplete lineage sorting (ILS). An

298 excess of either ABBA or BABA patterns is indicative of gene flow. Because of the low

299 resolution of many recent branches within major clades of the phylogenetic tree (see

300 Results), evidence for introgression was only evaluated between four well-supported 
301 major subclades, each of which is characterized by a distinct fruit color in our specific

302 dataset (i.e. the purple-, red-, and orange-fruited major clades, and a two-species clade of

303 green-fruited taxa; see Results). Patterson's $D$-statistic was calculated for all four-taxon

304 combinations including one taxon from the green-fruit lineage, one from red or orange-

305 fruit lineage, one from purple-fruit lineages, and one from tomato as the outgroup.

306 Patterson's $D$-statistic is calculated as (ABBA-BABA) / (ABBA+BABA) for biallelic

307 sites in the multiple sequence alignment (Durand et al., 2011; Green et al., 2010). To

308 further investigate the taxa involved in and the direction of introgression specifically

309 involving purple-fruited lineages, we also used a symmetric five-taxon phylogeny

310 method ' $D$-foil' test (Pease \& Hahn, 2015) on the transcriptome-wide concatenated

311 dataset, however the results of these analyses were inconclusive (see Supplementary text).

\section{Identifying genetic variation associated with trait evolution}

314 We used two general strategies to identify loci that might contribute to important

315 phenotypic trait (fruit and floral) transitions within Jaltomata. First, to identify loci that

316 have experienced lineage-specific de novo adaptive molecular evolution, we evaluated

317 loci for patterns of molecular evolution indicative of positive selection on specific

318 phylogenetic branches (i.e. $d_{N} / d_{S}>1$ ). Second, to identify variants that might have been

319 selected from segregating ancestral variation, we identified genetic variants that had

320 polyphyletic topologies that grouped lineages according to shared trait variation rather

321 than phylogenetic relationships ('PhyloGWAS'; Pease et al., 2016).

323 1) Lineage-specific de novo evolution associated with trait variation: We identified loci 
324 with signatures of de novo adaptive molecular evolution (i.e. significantly elevated rates

325 of non-synonymous substitution) across each available locus in our transcriptome

326 (sometimes called 'reverse ecology'; Li et al., 2008) as well as in a set of a priori

327 candidate loci identified based on known or putative functional roles associated with

328 floral or fruit trait variation (Krizek \& Anderson, 2013; Rausher, 2008; Specht \&

329 Howarth, 2015) (see supplementary text). Tests were only performed on the four best-

330 supported branches within the phylogeny (see Results). For each locus (group of

331 orthologs), we inferred putative adaptive evolution (i.e. $d_{N} / d_{S}>1$ ) using PAML v4.4

332 branch-site model (model = 2 and NS sites $=2$ ) on the target branches (Yang, 2007). In

333 each analysis, a likelihood ratio test (LRT) was used to determine whether the alternative

334 test model (fixed_omega = 0) was significantly better than the null model (fixed_omega

$335=1)$. In addition, because PAML uses a tree-based $d_{N} / d_{S}$ model to reconstruct ancestral

336 states and lineage-specific substitutions,_and because high levels of incongruence of gene

337 trees caused by ILS and introgression can produce misleading results when gene trees do

338 not match the assumed species tree (Mendes et al., 2016; Pease et al., 2016), we limited

339 our tests of molecular evolution to the subset of genes for which 1) the RAxML gene tree

340 contained the target ancestral branch (that is, the target branch was supported by the

341 genealogy of the tested/target locus); and 2) there was at least one non-synonymous

342 substitution that could be unambiguously assigned to this branch.

343 Prior to testing individual loci, we further filtered our data to ensure that poorly

344 aligned and/or error-rich regions were excluded from our alignments (as these tests are

345 particularly sensitive to alignment errors that generate spurious non-synonymous

346 changes). To do so, we used the program SWAMP v1.0 to remove regions from 
347 alignments when they showed higher than expected non-synonymous substitutions (i.e.

348 more than five non-synonymous substitutions in 15 codons; the second sliding-window

349 sequence alignment check, different from above) and a minimum sequence length of 50

350 codons (Harrison et al., 2014). We also required that each alignment must contain a

351 tomato sequence and orthologous sequences from all investigated Jaltomata species

352 (except for $J$. grandibaccata because reproductive tissues were not sampled from this

353 species)._The resulting sequence alignments were converted to codon-based MVF file

354 format (Pease \& Rosenzweig, 2015), prior to performing branch-site tests. We first

355 defined putative genes showing positive selection by using the uncorrected $p$-value $<0.01$

356 as cutoff. The false discovery rate (FDR; (Benjamini \& Hochberg, 1995) was then

357 calculated for the PAML $p$-values in each branch-specific test.

358 For the PAML analyses of a priori candidates, we used a slightly less stringent

359 statistical cutoff for consideration and required only that each cluster of orthologous

360 sequences had a minimum representation of species from each of the major clades (see

361 supplementary text); this allowed us to evaluate more of these loci while still testing

362 molecular evolution only on the four well-supported branches. For a priori genes

363 showing a significant signature of positive selection $(p<0.05)$ and for genes identified by

364 the genome-wide unbiased analyses (FDR $<0.1$ ), we manually checked the sequence

365 alignments to examine whether they contain putative multi-nucleotide mutations

366 (MNMs), which can cause false inferences of positive selection in the PAML branch-site

367 test (Venkat et al., 2017). Here we assigned an MNM in cases where we observed that a

368 single codon had 2 or 3 substitutions on the selected branch.

369 To determine the putative functional categories of genes with elevated per site 
370 non-synonymous substitution rates, and to assess whether these were enriched for

371 particular functional categories, genes with uncorrected $p$-value $<0.05$ from the

372 transcriptome-wide analysis were also examined using Gene Ontology (GO) terms. GO

373 term reference was obtained from the Gene Ontology project (www.geneontology.org).

374 GO terms for each gene were obtained from SolGenomics (ftp://www.solgenomics.net).

375 GO term enrichment analysis was performed with ONTOLOGIZER v2.0 using the

376 parent-child analysis (Bauer et al., 2008).

378 2) Ancestral genetic variation associated with trait variation: To identify shared ancestral

379 variants that were associated with trait variation across lineages, we used a 'PhyloGWAS'

380 approach (Pease et al., 2016) in which we searched for SNPs that were shared by current

381 accessions that share the same character state, regardless of their phylogenetic relatedness.

382 This approach is only informative in cases where trait variation is not confounded with

383 phylogenetic relationships, which in Jaltomata applies to floral shape transitions from the

384 ancestral 'rotate' form to the two derived forms (see Results). For our analysis, we treated

385 both campanulate and tubular corolla as the derived state, and rotate corolla as the

386 ancestral state (Figure S2). These categories of floral shape are also perfectly associated

387 with nectar color variation; species with rotate corollas have small amounts of clear/very

388 lightly colored nectar (ancestral), whereas species with campanulate or tubular corollas

389 have larger amounts of darkly colored red or orange nectar (derived). To assess whether

390 the number of nonsynonymous variants found to be associated with our defined groups of

391 floral traits (see Results) was greater than expected by chance, we generated a null

392 distribution due to ILS alone by simulating datasets over the species tree (Figure 2A) 
393 using the program $m s$ (Hudson, 2002). An associated $p$ value was determined by the

394 proportion of simulated datasets that have a greater number of genes perfectly associated

395 with the floral trait distribution than our observed value (see Supplementary text).

397 RESULTS

398 Transcriptome assembly and ortholog inference identified $>6000$ orthologs

399 In assembled transcriptomes from both reproductive and vegetative tissues for

400 each of 14 Jaltomata species (except for J. grandibaccata, which only included

401 vegetative tissues), the number of transcripts per lineage ranged from 46,841-132,050,

402 and mean transcript length ranged from 736-925 bp (Table S2). Based on our criteria for

403 ortholog identification (see Methods, Figure S1), we ultimately identified 6431 one-to-

404 one orthologous genes for which we had sequences from all 14 investigated Jaltomata

405 species and a unique tomato annotated coding sequence. All of these 6431 genes were

406 used in the concatenation, majority rule, or quartet-based phylogeny reconstructions.

407 From this dataset, we used 1517 genes in the BUCKy reconstruction (see Methods).

408 Since we did not sample RNA from the reproductive tissues of $J$. grandibaccata, we

409 excluded this species from analyses of locus-specific adaptive evolution; this resulted in a

410 slightly larger number of orthologs, including those expressed solely in flowers. The

411 resulting dataset had 6765 alignments of orthologous coding sequences, each containing

412 sequences from the remaining 13 Jaltomata species (with J. grandibaccata excluded) and

413 tomato. Among them, 4248 genes also have $C$. annuum orthologs, thus could also be used

414 to test for positive selection on the ancestral branch leading to Jaltomata. 
All four phylogenetic inference methods (concatenation, majority rule, quartet-

418 based, and Bayesian concordance) generated a nearly identical species tree topology

419 (Figure 2A, S3). In all trees, the first split in the species tree produces a well-supported

420 clade that includes three north- and central-American species (J. procumbens, J.

421 repandidentata, and J. darcyana) that all share floral traits (rotate corollas and light

422 nectar) and make dark purple/purple fruit $(\mathrm{CF}=87)$. The remaining 11 species, that are

423 found exclusively in South America and vary in floral traits and fruit colors, form a single

424 moderately supported clade $(\mathrm{CF}=66)$. Based on sequence divergence at synonymous sites

425 (Table S3), lineages within the non-purple-fruited clade have pairwise distance of 0.26\%

426 to $0.53 \%$, and differ from the purple-fruited lineages by $0.95 \%$ to $1.29 \%$. Within the non-

427 purple-fruited group, our reconstruction indicates that the red-fruited species J. auriculata

428 is sister to the remaining species. The remaining species are split into a moderately

429 supported clade $(\mathrm{CF}=67)$ of two species (J. calliantha and J. quipuscoae) that share floral

430 traits and green fruits, and a relatively poorly supported clade $(\mathrm{CF}=19)$ consisting of the

431 remaining eight species that vary extensively in floral traits but all produce orange fruit.

432 Although quantitative support for different clades varied based on inference method

433 (Figure S3), all trees produced the same topology, with the exception of inferred

434 relationships within the orange-fruited group among J. biflora, J. sinuosa, J. aijana and J.

435 umbellata. In that case, all concatenation, quartet-based, and concordance trees supported

$436 J$. sinuosa as more closely related to J. aijana and J. umbellata (6\% of gene trees support

437 this grouping), while consensus trees placed J. sinuosa as sister to J. biflora (7\% of gene

438 trees support this grouping). 
$440 \quad$ Segregating variation is broadly shared among species in different subclades

441 To quantify how much variation shared among present subclades—presumably

442 because of either shared ancestral variation or ongoing introgression-we mapped RNA-

443 seq reads from each species to the tomato reference genome and called high-quality

444 variants from $\sim 8$ million sites with more than $10 \mathrm{X}$ sequencing depth for all investigated

445 species. We identified a large number of sites that are sorting the same allele among

446 different subclades. Among them, 4303 variant sites are sorting in all four subclades

447 (Figure 3A). We also quantified how many sites that are heterozygous in one lineage

448 (accession) have the same two alleles sorting in other subclades. Within each lineage, the

449 proportion of heterozygous sites range from $0.02 \% \sim 0.16 \%$ (Table S4), which is

450 comparable to the level of heterozygosity observed in self-compatible tomato species

451 (Pease et al., 2016). For $13.57 \%$ to $64.35 \%$ of heterozygous sites in one species (Figure

452 3A, Table S4), both alleles could also be identified in other subclades, again indicative of

453 a large amount of shared allelic variation.

$\underline{\text { Phylogenomic discordance accompanies rapid diversification }}$

$456 \quad$ As expected given the large number of genes $(n=6431$ and 6,223,350 sites in

457 total) used for phylogenetic inference, our resulting species trees had very strong

458 bootstrap support for almost all nodes (Figure S3A and S3C). Despite this,

459 reconstructions also revealed evidence of extensive gene tree discordance consistent with

460 rapid consecutive lineage-splitting events in this group (Figure 2B; S3B and S3D). For

461 instance, the 6431 genes inferred 6431 different topologies, none of which matched the 
462 topology of the inferred species tree (Figure 2A). The concatenation tree has many

463 extremely short internal branches, where gene trees show high levels of phylogenetic

464 discordance. We detected a strong correlation between the internal branch length and

465 levels of discordance $(P=0.0001$, Figure S4), consistent with both ILS and introgression.

466 Short branch lengths and extensive discordance were also detected for trees built

467 individually for each of the 12 chromosomes (Figure S5; supplementary text).

468 Only three branches within Jaltomata are supported with relatively little

469 discordance, i.e., with Bayesian CFs greater than 50 (Figure S3D): the branch leading to

470 the purple-fruited clade, the branch uniting all non-purple-fruit Jaltomata lineages, and

471 the branch leading to the two green-fruited lineages (Figure 2A). Along with the ancestral

472 Jaltomata branch, these were the four branches on which most of our subsequent

473 analyses were performed.

Introgression after speciation among major clades of Jaltomata lineages

Given the apparent high level of phylogenetic discordance among our examined

477 species, we tested for evidence of introgression on the background of presumed ILS. To

478 do so, we calculated genome-wide $D$-statistics using the ABBA-BABA test. We only

479 examined introgressions across well-supported subclades. In particular, we compared the

480 distribution of trees in which one of two sister taxa (here, a species from either the red-,

481 green-, or orange-fruited lineage) is closer to more distantly related species (in the purple-

482 fruited clade) than the other. We found several such cases (Figure 3B). For example,

483 there was a significant excess of sites that grouped the red-fruited lineage (J. auriculata)

484 with a purple-fruited lineage, relative to the number of sites that grouped the green- 
485 fruited lineage with the purple-fruited lineage, indicative of detectable gene flow between

486 the red-fruited and purple-fruited lineages since their split (Figure 3B and Table S5). We

487 also inferred putative introgression, in at least two separate events, involving six species

488 in the orange-fruited clade with the purple-fruited clade (Figure 3B and Table S5). First,

489 we inferred a shared introgression event between the purple-fruited group and three of the

490 orange-fruited species (J. grandibaccata, J. dendroidea, and J. incahuasina); this excess

491 includes shared specific sites that support the same alternative tree topology for each of

492 these three ingroup species, suggesting that it likely involved the common ancestor of all

493 three contemporary orange-fruited species (Table S6). Second, we detected evidence for

494 gene flow between the remaining orange-fruited species (J. yungayensis, J. biflora and $J$.

495 sinuosa) and the purple-fruited lineage, in the form of significant genome-wide $D$ -

496 statistics (Table S5). Because we did not observe an excess of shared specific sites

497 supporting the same alternative tree topology among these three orange-fruited species

498 (Table S6), these patterns are suggestive of three putative independent introgression

499 events. However, given very low resolution of patterns of relatedness among orange-

500 fruited species, the specific timing of these events is hard to resolve.

502 Ancestral state reconstruction suggests different histories for fruit color and floral trait

503 evolution

504 Based on the inferred species tree (Figure 2A), we reconstructed the ancestral

505 states of fruit and floral traits (Figure 4; S6). The four subclades of Jaltomata species

506 were inferred to have evolved different fruit colors at their corresponding common

507 ancestors (Figure 4A). Our reconstruction suggests that the derived nectar traits 
508 (orange/red nectar color, and increased nectar volume) probably evolved at the common

509 ancestor of the green/orange-fruited clade (Figure S6A and S6B), with two subsequent

510 reversions to ancestral conditions within this clade. The evolution of the two derived

511 corolla shapes in Jaltomata (campanulate and tubular) appears to be more complex

512 (Figure 4B). At the majority of internodes within the non-purple-fruited lineages, all three

513 corolla shapes (i.e. rotate (ancestral), campanulate and tubular) show $\geq 10 \%$ probability of

514 being the ancestral state, making specific inferences about corolla shape evolution within

515 this clade uncertain. Consistent with these patterns, we found that concordance factors

516 were very low at almost all internodes within the radiating subgroup that displays the

517 derived floral traits (i.e., the non-purple-fruited lineages) (Figure 2A; S3D), whereas they

518 were considerably higher on branches associated with fruit color evolution (including the

519 branch uniting the two green-fruited species analyzed).

520 These analyses suggest alternative evolutionary and genetic histories for our traits

521 of interest. In particular, strong associations between fruit color transitions and specific

522 branches/clades within Jaltomata suggests that the underlying genetic changes are more

523 likely due to conventional lineage-specific de novo evolution along the relevant branches.

524 In contrast, the distribution of floral trait variation produces an ambiguous reconstruction

525 of trait transitions, especially for floral shape, such that the distribution of ancestral

526 versus derived floral shape variation is unassociated with phylogenetic relationships in

527 the non-purple-fruited Jaltomata clade (Figure 4B). While strictly de novo evolution

528 occurring multiple times is not excluded as an explanation of floral evolutionary

529 transitions, one alternative is that these trait transitions drew upon shared variation

530 segregating in the ancestor of these lineages. Accordingly, in the next sections we 
531 evaluate both lineage-specific de novo evolution and selection from standing ancestral

532 variation when searching for genetic variants that might have contributed to floral trait

533 evolution. The lineage-specific de novo evolution analysis alone is used to identify

534 potential candidates for fruit color evolution, since the approach we use to identify

535 standing ancestral variation is only informative in cases where trait variation is not

536 confounded with phylogenetic relationship.

Loci with patterns of positive selection associated with lineage-specific trait evolution

539 We performed tests of molecular evolution for all orthologous clusters that

540 contained a sequence from every Jaltomata accession and an ortholog from the tomato

541 outgroup. Depending upon the specific branch being tested, we detected evidence for

542 positive selection in $\sim 1-2 \%$ of loci in our dataset, based on whether the locus had

543 significantly elevated $d_{N} / d_{S}$ ratios $\left(d_{N} / d_{S}>1 ; p<0.01\right)$. This included $1.88 \%$ of genes $(67$

544 out of 3556 testable genes; Table S7) on the Jaltomata ancestral branch, $1.58 \%$ in the

545 purple-fruited group (48 out of 3033 testable genes; Table S8), 2.61\% in the red-fruited

546 group (70 out of 2686 testable genes; Table S9), 1.96\% in the green-fruited group (30 out

547 of 1531 testable genes; Table S10), and $0.74 \%$ in the non-purple-fruited Jaltomata

548 lineages (15 out of 2039 testable genes; Table S11). Many of the genes showing elevated

$549 d_{N} / d_{S}$ appear to have general molecular functions (e.g. transcription, protein synthesis, or

550 signaling), including numerous genes involved in various stress responses, such as heavy

551 metal tolerance, sugar starvation response, protection from ultraviolet (UV) radiation and

552 extreme temperature, and herbivore and pathogen resistance (Table S7-11). Our

553 positively selected loci contain genes functionally associated with photosynthesis, 
554 fatty/lipid biosynthesis and transportation, and sugar signal transduction (observed in the

555 GO enrichment analysis; Table S12-16), as well as loci with unknown functions.

After controlling for multiple tests using an FDR $<0.1$ on each branch tested, only

557 three genes on the Jaltomata ancestral branch, one gene on the purple-fruited ancestral

558 branch, and four genes on the red ancestral branch, remained significant for $d_{N} / d_{S}>1$.

559 Interestingly, for 5 of these 8 loci, the inference of positive selection appears to be due to

560 the presence of a multi-nucleotide mutation (MNM) specifically on the target branch, a

561 mutational pattern known to produce spurious inferences of positive selection in PAML's

562 branch-site test (Venkat et al., 2017). With one exception, all of these potential false

563 positives were found on the ancestral Jaltomata or purple-fruited clade branches, the

564 longest Jaltomata-specific branches in our analyses; as MNMs are more likely to appear

565 on long branches (they are relatively rare mutations) these are expected to be enriched for

566 these spurious inferences in the branch-site test (Venkat et al., 2017). Our three remaining

567 loci, with positive selection on the red-fruited branch, include a gene (BANYULS;

568 ortholog to Solyc03g031470) with functional roles in pigmentation (see Discussion).

569 We also detected several instances where slightly less stringent criteria $\left(d_{N} / d_{S}>1\right.$;

$570 p<0.05)$ revealed lineage-specific adaptive evolution of our a priori candidate genes

571 occurring on a branch that is also inferred to be associated with the evolution of derived

572 traits (Table S17). Most notably, we found evidence of positive selection on candidate

573 loci that are likely to be involved in fruit color, including a gene encoding $\zeta$-carotene

574 isomerase (Z-ISO; ortholog to Solyc12g098710) on the red-fruited lineage, and the two

575 other genes significant on the ancestral branch of the green-fruited lineages encoding

576 carotenoid cleavage enzyme 1A (CCD1A; ortholog to Solyc01g087250) and zeaxanthin 
577 epoxidase (ZEP; ortholog to Solyc02g090890) (Figure 5, see Discussion). We detected

578 signatures of positive selection on fewer of the genes involved in floral development,

579 mostly notably in the MADS-box gene APETALA3 (AP3/DEF, ortholog to

580 Solyc04g081000) on the ancestral branch to the purple-fruited lineage. Overall, we note

581 that many of our loci (including a priori candidates) did not meet the requirements to be

582 tested for positive selection (Table S7); in particular, gene trees for many loci lacked the

583 required support for a specific internal branch, either because of incongruence or limited

584 phylogenetic signal, especially within the rapidly diverging orange-fruited clade (Table

585 S17).

586

$587 \quad$ Loci potentially associated with trait evolution from standing ancestral variation

588 To investigate whether ancestral variants are potentially associated with floral

589 trait diversification, we performed a "PhyloGWAS" analysis (Pease et al., 2016). Such

590 variants will have differentially fixed among descendant lineages, leading to genes that

591 cluster species together based on floral traits regardless of their overall phylogenetic

592 relationships. We found 31 genes with nonsynonymous variants perfectly associated with

593 the derived floral traits (Table S18), which was significantly more than the number of

594 loci expected by chance to have segregation patterns that exactly match the tip states $(p<$

$\left.5959.3 \times 10^{-5}\right)$. Most of these genes are characterized by only one or few nucleotide

596 differences, which is an expected pattern for variants recently selected from standing

597 ancestral variation (Pease et al., 2016). These results suggest that one or few molecular

598 variants present in ancestral populations could contribute to the multiple apparent

599 transitions to derived floral shapes in Jaltomata. Among the loci identified by our 
600 approach, some genes are potentially functionally related to petal development, including

601 ARGONAUTE1 (AGO1) and xyloglucan endotransglucosylase/hydrolase 2 (DcXTH2)

602 (see Discussion).

603

604 DISCUSSION

605 Within rapidly radiating groups, the patterns of genetic relatedness among

606 lineages provide essential data for determining the pace and location of important trait

607 transitions, and their underlying genes; both are critical for understanding the drivers of

608 rapid diversification and speciation. Our phylogenomic analyses of the 14 investigated

609 Jaltomata species revealed genome-wide gene tree discordance, and a highly complex

610 history of genetic relatedness among contemporary lineages, consistent with other studies

611 of recently radiating groups (Brawand et al., 2014; Garrigan et al., 2012; Novikova et al.,

612 2016; Pease et al., 2016). We identified substantial ILS and shared ancestral

613 polymorphism, as well as evidence of putative introgressions among the subclades of

614 Jaltomata species, as the sources of this observed complex genome-wide history. This

615 complexity was also reflected in inferences about the evolution of major trait transitions

616 within the group. We found differences in the patterns of fruit versus floral character

617 evolution and in our inferred confidence in the reconstruction of these patterns, including

618 their likely risk of hemiplasy. Given this, we used several strategies to identify loci that

619 might contribute to the evolution of these traits, including examining lineage-specific de

620 novo adaptive evolution along well-supported branches and identifying variants that

621 might have been selected from standing ancestral variation. Overall, by combining

622 evidence from molecular evolution with data on trait variation across a clade — and a 
623 more direct accounting for the risk of hemiplasy — we generated more conservative, but

624 credible, inferences of candidate genes responsible for the evolution of ecologically

625 important phenotypic traits. Specifically, we identified several functionally relevant

626 candidate genes for our target trait transitions, and different potential sources of adaptive

627 evolution fueling changes in target floral versus fruit traits.

Extensive ILS and several introgression events produce a complex genome-wide history

630 during rapid diversification

Our reconstruction of phylogenetic relationships based on a transcriptome-wide

632 dataset agrees with previous studies that identified three major Jaltomata sub-clades

633 primarily distinguished from each other by their fruit colors (Miller et al., 2011; Särkinen

634 et al., 2013), including the previous inference that the clade of purple-fruited species is

635 sister to the rest of the genus (Särkinen et al., 2013). However, the relationships among

636 species within each subclade show high levels of discordance, and the distribution of

637 genetic variation strongly indicates a rapid and recent evolutionary origin; the internal

638 branch lengths within each subclade are short, especially in the non-purple-fruited

639 lineages (Figure 2A). Accordingly, the history of these species_including the

640 orange/green-fruited lineages that show the most floral trait diversity among Jaltomata

641 species - is expected to be strongly affected by ILS and introgression.

642 Indeed our genome-wide reconstruction indicated that, although concatenation or

643 quartet-based approaches generated a species tree with high bootstrap support values

644 (commonly observed when inferring trees from large amounts of data (Kubatko \&

645 Degnan, 2007; Salichos \& Rokas, 2013), gene tree discordance was rampant, and 
646 individual gene trees showed highly variable support for the specific placement of

647 individual species (Figure 2B) especially at short branches (Figure 2A). Our finding of

648 extensive genome-wide ILS in the genus Jaltomata agrees with other recent studies on

649 contemporary (Novikova et al., 2016; Pease et al., 2016) and relatively ancient adaptive

650 radiations in plant species (Wickett et al., 2014; Yang et al., 2015), and is emerging as a

651 universal signal of rapid radiation in comparative genome-wide datasets.

653 subclade was insufficiently clear to investigate introgression within subgroups. However,

654 across major sub-clades we identified at least two clear introgression events that involved

655 either orange and red-fruited lineages with the purple-fruited lineages, similar to the

656 detection of introgression events in other recent genome-wide studies on closely related

657 plant species (Eaton \& Ree, 2013; Novikova et al., 2016; Owens et al., 2016; Pease et al.,

658 2016). Interestingly, in one case an excess of sites supported a shared introgression

659 pattern between three orange-fruited species (i.e. J. grandibaccata, J. dendroidea, and J.

660 incahuasina) and the purple-fruited clade, consistent with a scenario in which

661 introgression involved the recent common ancestor of these three orange-fruited species.

662 Moreover, in this case, the inference of a single shared introgression event itself provided

663 more confidence in this specific ancestral branch within the orange-fruited clade. Overall,

664 as with ILS, post-speciation introgression is another inference frequently emerging from

665 contemporary phylogenomic studies of radiations.

666

667 Inferring the history of trait evolution and the contributing loci in the presence of

668 rampant discordance 
670 for inferences of trait and gene evolution. When ILS or introgression can plausibly

671 explain the discordant distribution of traits, it might be impossible to infer trait evolution

672 with any certainty in the absence of additional independent information about target traits,

673 such as their genetic basis (Hahn \& Nakhleh, 2016). In contrast, the evolutionary

674 transitions of some traits might be confidently inferred as long as the relevant branches

675 and resulting relationships are associated with higher levels of concordance (Hahn \&

676 Nakhleh, 2016). Species in Jaltomata exhibit extensive trait diversity, most notably in

677 fruit color, corolla shape, and nectar volume and color (Figure 2A) (Miller et al., 2011),

678 and one of the main goals in this study was to better understand the evolutionary history

679 of these trait transitions, including the genetic basis of the traits associated with rapid

680 phenotypic diversification. A previous phylogenetic study based on a single locus

681 suggested that floral traits (including corolla shape and nectar color) might have evolved

682 multiple times independently in Jaltomata species (Miller et al., 2011). However, the

683 presence of rampant discordance, and abundant evidence of shared ancestral variation,

684 makes inferring the history of trait transitions and their genetic basis especially

685 challenging in this group. Indeed, our analyses indicated that different classes of trait

686 transition — most notably fruit color versus floral shape variation-were differently

687 susceptible to hemiplasy. For floral shape evolution in Jaltomata, a lack of resolution and

688 high gene tree discordance at key nodes within the phylogeny, including within the clade

689 displaying the greatest phenotypic diversity (Figure 2A and Figure 4B), mean that

690 hemiplasy is a plausible explanation of the discordant distribution of similar floral

691 traits-rather than multiple independent evolutionary events (i.e. homoplasy). In contrast, 
692 we showed that the history of fruit color evolution could be confidently inferred, as these

693 trait transitions occurred on branches with higher levels of concordance and therefore

694 lower risks of hemiplasy (Figure 2A and Figure 4A).

695 Accounting for the potential influence of hemiplasy is also critical when

696 generating hypotheses about the loci that could have contributed to trait transitions. In

697 general, incorrect reconstructions of trait history will suggest incorrect candidates

698 involved in the evolution of those traits. Moreover, tests of molecular evolution can be

699 specifically misled if trait transitions occur on discordant gene trees (Mendes et al., 2016).

700 Accordingly, to identify loci that might be responsible for any particular trait transitions,

701 different approaches will be appropriate depending upon the confidence with which

702 hemiplasy can be excluded or not. For traits evolving on branches where discordance is

703 low, confidence is high, and hemiplasy is unlikely, it is reasonable to expect that lineage-

704 specific de novo substitutions are a substantial contributor to relevant trait evolution.

705 Given a high risk of hemiplasy, genetic variation underpinning trait evolution could

706 potentially come from additional sources, including recruitment of ancestral

707 polymorphisms and/or introgression. These differences are exemplified in our study by

708 the alternative histories, and different genetic hypotheses, generated for fruit color versus

709 floral shape traits.

710

711 Floral shape evolution - Based on the inferred species tree (Figure 2A), the 14

712 investigated Jaltomata species are not related according to their floral shape traits (i.e.

713 floral shape is distributed paraphyletically). Moreover, the branch lengths leading to

714 lineages with derived character states are uniformly short with high levels of gene tree 
715 discordance (Figure 2A), so that the probability of hemiplasy is expected (Hahn \&

716 Nakhleh, 2016) to be very high. Indeed, when we reconstruct the evolution of corolla

717 shape (Figure 4B), the three alternative corolla morphs were inferred to be almost equally

718 likely at the common ancestor of non-purple-fruited lineages. It is possible that this lack

719 of resolution is due to introgression. For example, the ancestral floral character states (i.e.

720 rotate corolla shapes and clear nectar) found in J. yungayensis and J. sinuosa within the

721 orange-fruited clade could be due to alleles introgressed from purple-fruited species, as

722 we identified putative introgression events between those lineages (Figure 3B). However,

723 the lack of a reference genome for Jaltomata precluded us from more directly

724 investigating evidence (for example, locus-specific patterns of introgression) that

725 introgression might contribute to the distribution of ancestral floral traits within this sub-

726 clade. Instead, because of the high risk of hemiplasy and low resolution of ancestral states,

727 we used several approaches to identify the genetic variants associated with the two other

728 potential sources of trait variation.

First, if the paraphyletic distribution of derived traits is due to hemiplasy among

730 species, the relevant nucleotide differences should be at the same sites in all lineages that

731 share derived traits (Hahn \& Nakhleh, 2016). Using this rationale, Pease et al. (2016)

732 identified tens to hundreds of genetic variants among wild tomato lineages that were

733 exclusively associated with each of three ecological factors, variants that are candidate

734 targets of parallel ecological selection on standing ancestral variation in that group. Here,

735 we used an analogous approach to look for variants associated with phenotypic (floral)

736 trait variation in Jaltomata, and identified 31 candidate genes with nonsynonymous

737 variants that are completely correlated with the distribution of floral trait variation 
738 (derived vs. ancestral; Figure S2). Among them, the gene AGO1 (ortholog to

739 Solyc02g069260) is known to be necessary in Arabidopsis floral stem cell termination

740 and might act through $C U C 1$ and $C U C 2$ (a priori candidate genes), which redundantly

741 specify boundaries of floral meristem (Ji et al., 2011; Kidner \& Martienssen, 2005).

742 Another gene DcEXPA2 (ortholog to Solyc02g091920) is known to be markedly up-

743 regulated in the petals of carnation (Diathathus caryophyllus), and is potentially

744 associated with the petal growth and development (Harada et al., 2010). We also note that,

745 although these shared hemiplasious variants could be due to post-speciation introgression

746 rather than sorting from ancestral variation, given the often allopatric geographical

747 distribution of our lineages (Figure 1) and evidence of substantial allelic sharing among

748 contemporary subclades (Figure 3A), we infer that these variants were more likely sorted

749 from ancestral variation.

750 Second, because our reconstruction of floral trait evolution could not exclude the

751 role of lineage-specific de novo mutation, we also examined our transcriptomes for genes

752 showing lineage-specific evolution associated with the derived floral traits. However, we

753 found that only a small number of genes were testable due to the extensive gene tree

754 discordance specifically at the branches leading to subclades with the derived floral

755 characters. Moreover, none of our a priori candidate genes involved in floral

756 development showed suggestive patterns of molecular evolution on internal branches of

757 Jaltomata (Table S7), nor did we find any other functionally suggestive (floral

758 development-related) genes adaptively evolving on branches leading to specific

759 subclades within Jaltomata, with the exception of APETALA3 (AP3) - a gene associated

760 with the formation of petals and stamens in flowering plants, including 
761 Arabidopsis(Wuest et al., 2012; Specht \& Howarth, 2015)_although this was detected

762 on the branch leading to the purple-fruited clade, within which all species retain the

763 ancestral rotate corolla form.

764

765 Fruit color evolution - In contrast to floral traits, ancestral state reconstruction in the 14

766 investigated Jaltomata species suggested that fruit color transitions follow phylogenetic

767 relationships. The ancestral states of fruit colors at most relevant internodes can be

768 inferred with high confidence (Figure 4A), and the internal branches leading to fruit trait

769 transitions are well-supported, indicating a low probability of hemiplasy (Figure 2A).

770 Accordingly, we identified a set of loci showing adaptive evolution specifically on these

771 branches, provided that each tested gene tree 1) supported the internal branch being

772 tested, and 2) showed at least one clade-specific nonsynonymous substitution on that

773 branch (Mendes et al., 2016; Pease et al., 2016), to avoid potential inference errors

774 associated with examining genes that have topologies discordant with the species tree

775 (Mendes et al., 2016). Our analyses revealed multiple adaptively evolving candidate loci

776 with clear functional relevance to these trait transitions, including several a priori

777 candidate genes involved in the carotenoid pathway (Yuan et al., 2015) (Figure 7). First,

778 Z-ISO (ortholog to Solyc12g098710), a key enzyme in the production of red-colored

779 lycopene in the carotenoid biosynthetic pathway (Chen et al., 2010), was positively

780 selected on the branch leading to our one species with bright red fruit, J. auriculata.

781 Interestingly, this gene was also previously found to show adaptive evolution specifically

782 on the branch leading to the red-fruited (Esculentum) group in wild tomatoes (Pease et

783 al., 2016). Second, on the branch leading to our two green-fruited species, we found 
784 significantly elevated $d_{N} / d_{S}$ ratios for both CCD1A (ortholog to Solyc01g087250), a gene

785 whose product participates in the conversion of carotenoid pigments to isoprenoid

786 volatiles (Ilg et al., 2014), and for ZEP (ortholog to Solyc02g090890), which converts

787 zeaxanthin to violaxanthin (Marin et al., 1996). CCD1 has previously been identified in

788 tomato fruits as responsible for generating flavor volatiles (Auldridge et al., 2006; Simkin

789 et al., 2004). This functional observation from a closely related group is intriguing

790 because fruits of our two green-fruited species (J. quipuscoae and J. calliantha) appear to

791 produce the strongest scent within the 14 Jaltomata species analyzed here (J. Kostyun,

792 unpubl. data). This apparent increase in fragrance-presumably due to changed volatile

793 organic compounds — might play a role in attracting vertebrate frugivores for seed

794 dispersal.

795 In addition to carotenoids, among a priori candidate genes involved in the

796 biosynthesis pathway of water-soluble vacuolar anthocyanin pigments, we detected

797 BANYULS (ortholog to Solyc03g031470) selected on the red-fruited branch. (Note that

798 PAML also indicates adaptive molecular evolution of this locus on the purple-fruited

799 branch, but this appears to be due to the presence of a MNM; see results). In addition to $a$

800 priori candidates, genome-wide unbiased analyses also identified that multiple genes

801 belonging to $R 2 R 3 M Y B, B H L H$ and $W D 40$-repeats classes of loci were under positive

802 selection in purple-fruited lineages and the red-fruited lineage. The $M Y B-B H L H-W D 40$

803 TF complexes are known to regulate cellular differentiation pathways, including of the

804 epidermis, as well as transcription of anthocyanin structural genes (Gonzalez et al., 2008;

805 Jaakola, 2013; Ramsay \& Glover, 2005). 

in rapid radiating lineages

As highlighted here and in numerous recent phylogenomic studies (Eaton \& Ree,

810 2013; Novikova et al., 2016; Owens et al., 2016; Pease et al., 2016), both ILS and

811 introgression contribute to the history of diversification within radiating clades, so that

812 evolution in these groups is more complex than can be represented by a simple

813 bifurcating species tree. This complexity has important implications for empirical

814 inferences about historical relationships and trait evolution, because assuming resolved

815 relationships without taking into account incongruence can fundamentally mislead

816 inferences in both these cases (Hahn \& Nakhleh, 2016). It also must be accounted for

817 when considering the genetic changes that might have fueled diversification; when a trait

818 has several possible alternative evolutionary histories, it is necessary to investigate the

819 range of alternative sources of genetic variation-including de novo lineage-specific

820 evolution and selection from ancestral variation - that could fuel this trait evolution.

821 Here, we provided a genome-wide analysis of the recently diversified plant genus

822 Jaltomata in which we consider the relative risk of hemiplasy while identifying

823 candidates for the specific loci underlying trait evolution. Our analysis highlights a

824 growing appreciation that rapid radiations can and likely do draw on multiple sources of

825 genetic variation (Hedrick, 2013; Pease et al., 2016; Richards \& Martin, 2017). Indeed,

826 while independently originating variants could explain the recurrent evolution of

827 phenotypic similarity — a frequent observation in adaptive radiations - it is clear that

828 shared ancestral genetic variation, or alleles introgressed from other lineages, have also

829 made substantial contributions (Elmer \& Meyer, 2011; Stern, 2013). Going forward, it 
830 will be necessary to distinguish between these alternative scenarios to understand how

831 different evolutionary paths contribute to phenotypic convergence and differentiation

832 (Martin \& Orgogozo, 2013; Stern, 2013) and to identify the specific variants responsible.

\section{ACKNOWLEDGEMENTS}

The authors thank James Pease, Rafael Guerrero and Fabio Mendes for advice on performing comparative phylogenomic and molecular evolution analyses. This research was funded by National Science Foundation grant DEB-1135707, to LCM and MWH.

\section{AUTHOR CONTRIBUTIONS}




\section{LITERATURE CITED}

Auldridge, M. E., McCarty, D. R., \& Klee, H. J. (2006). Plant carotenoid cleavage oxygenases and their apocarotenoid products. Current Opinion in Plant Biology, 9, 315-321.

Avise, J. C., \& Robinson, T. J. (2008). Hemiplasy: a new term in the lexicon of phylogenetics. Systematic Biology, 57, 503-507.

Bauer, S., Grossmann, S., Vingron, M., \& Robinson, P. N. (2008). Ontologizer 2.0-a multifunctional tool for GO term enrichment analysis and data exploration. Bioinformatics, 24, 1650-1651.

Benjamini, Y., \& Hochberg, Y. (1995). Controlling the false discovery rate: a practical and powerful approach to multiple testing. Journal of the Royal Statistical Society. Series B (Methodological), 289-300.

Bouckaert, R. R. (2010). DensiTree: making sense of sets of phylogenetic trees. Bioinformatics, 26, 13721373.

Brawand, D., Wagner, C. E., Li, Y. I., Malinsky, M., Keller, I., Fan, S., . . Bezault, E. (2014). The genomic substrate for adaptive radiation in African cichlid fish. Nature, 513, 375-381.

Chen, Y., Li, F., \& Wurtzel, E. T. (2010). Isolation and characterization of the Z-ISO gene encoding a missing component of carotenoid biosynthesis in plants. Plant Physiology, 153, 66-79.

Cui, R., Schumer, M., Kruesi, K., Walter, R., Andolfatto, P., \& Rosenthal, G. G. (2013). Phylogenomics reveals extensive reticulate evolution in Xiphophorus fishes. Evolution, 67, 2166-2179.

Degnan, J. H., \& Rosenberg, N. A. (2009). Gene tree discordance, phylogenetic inference and the multispecies coalescent. Trends in Ecology \& Evolution, 24, 332-340.

Dobin, A., Davis, C. A., Schlesinger, F., Drenkow, J., Zaleski, C., Jha, S., . . Gingeras, T. R. (2013). STAR: ultrafast universal RNA-seq aligner. Bioinformatics, 29, 15-21.

Durand, E. Y., Patterson, N., Reich, D., \& Slatkin, M. (2011). Testing for ancient admixture between closely related populations. Molecular Biology and Evolution, 28, 2239-2252.

Eaton, D. A., \& Ree, R. H. (2013). Inferring phylogeny and introgression using RADseq data: an example from flowering plants (Pedicularis: Orobanchaceae). Systematic Biology, 62, 689-706.

Elmer, K. R., \& Meyer, A. (2011). Adaptation in the age of ecological genomics: insights from parallelism and convergence. Trends in Ecology \& Evolution, 26, 298-306.

Felsenstein, J. (1985). Phylogenies and the comparative method. The American Naturalist, 125, 1-15.

Fontaine, M. C., Pease, J. B., Steele, A., Waterhouse, R. M., Neafsey, D. E., Sharakhov, I. V., . . Kakani, E. (2015). Extensive introgression in a malaria vector species complex revealed by phylogenomics. Science, 347, 1258524.

Fu, L., Niu, B., Zhu, Z., Wu, S., \& Li, W. (2012). CD-HIT: accelerated for clustering the next-generation sequencing data. Bioinformatics, 28, 3150-3152.

Garrigan, D., Kingan, S. B., Geneva, A. J., Andolfatto, P., Clark, A. G., Thornton, K. R., \& Presgraves, D. C. (2012). Genome sequencing reveals complex speciation in the Drosophila simulans clade. Genome Research, 22, 1499-1511.

Gonzalez, A., Zhao, M., Leavitt, J. M., \& Lloyd, A. M. (2008). Regulation of the anthocyanin biosynthetic pathway by the TTG1/bHLH/Myb transcriptional complex in Arabidopsis seedlings. The Plant Journal, 53, 814-827.

Grabherr, M. G., Haas, B. J., Yassour, M., Levin, J. Z., Thompson, D. A., Amit, I., . . Zeng, Q. (2011). Trinity: reconstructing a full-length transcriptome without a genome from RNA-Seq data. Nature Biotechnology, 29, 644.

Green, R. E., Krause, J., Briggs, A. W., Maricic, T., Stenzel, U., Kircher, M., . . Fritz, M. H.-Y. (2010). A draft sequence of the Neandertal genome. Science, 328, 710-722.

Haas, B. J., Papanicolaou, A., Yassour, M., Grabherr, M., Blood, P. D., Bowden, J., . . Lieber, M. (2013). De novo transcript sequence reconstruction from RNA-seq using the Trinity platform for reference generation and analysis. Nature Protocols, 8, 1494-1512.

Hahn, M. W., \& Nakhleh, L. (2016). Irrational exuberance for resolved species trees. Evolution, 70, 7-17.

Harada, T., Torii, Y., Morita, S., Onodera, R., Hara, Y., Yokoyama, R., . . . Satoh, S. (2010). Cloning, characterization, and expression of xyloglucan endotransglucosylase/hydrolase and expansin genes associated with petal growth and development during carnation flower opening. Journal of Experimental Botany, erq319.

Harrison, P. W., Jordan, G. E., \& Montgomery, S. H. (2014). SWAMP: sliding window alignment masker for PAML. Evolutionary Bioinformatics, 10, 197. 
Heath, T. A., Hedtke, S. M., \& Hillis, D. M. (2008). Taxon sampling and the accuracy of phylogenetic analyses. Journal of Systematics and Evolution, 46, 239-257.

Hedrick, P. W. (2013). Adaptive introgression in animals: examples and comparison to new mutation and standing variation as sources of adaptive variation. Molecular Ecology, 22, 4606-4618.

Hudson, R. R. (2002). Generating samples under a Wright-Fisher neutral model of genetic variation. Bioinformatics, 18, 337-338.

Huelsenbeck, J. P., \& Ronquist, F. (2001). MRBAYES: Bayesian inference of phylogenetic trees. Bioinformatics, 17, 754-755.

Ilg, A., Bruno, M., Beyer, P., \& Al-Babili, S. (2014). Tomato carotenoid cleavage dioxygenases 1A and 1B: Relaxed double bond specificity leads to a plenitude of dialdehydes, mono - apocarotenoids and isoprenoid volatiles. FEBS Open Bio, 4, 584-593.

Jaakola, L. (2013). New insights into the regulation of anthocyanin biosynthesis in fruits. Trends in Plant Science, 18, 477-483.

Ji, L., Liu, X., Yan, J., Wang, W., Yumul, R. E., Kim, Y. J., . . Zheng, B. (2011). ARGONAUTE10 and ARGONAUTE1 regulate the termination of floral stem cells through two microRNAs in Arabidopsis. PLoS Genetics, 7, e1001358.

Kidner, C. A., \& Martienssen, R. A. (2005). The role of ARGONAUTE1 (AGO1) in meristem formation and identity. Developmental Biology, 280, 504-517.

Knapp S. (2010). On 'various contrivances': pollination, phylogeny and flower form in the Solanaceae. Philosophical Transactions of the Royal Society B, 365, 449-460.

Kostyun, J. L., \& Moyle, L. C. (2017). Multiple strong postmating and intrinsic postzygotic reproductive barriers isolate florally diverse species of Jaltomata (Solanaceae). Evolution.

Krizek, B. A., \& Anderson, J. T. (2013). Control of flower size. Journal of Experimental Botany, 64, 14271437.

Kubatko, L. S., \& Degnan, J. H. (2007). Inconsistency of phylogenetic estimates from concatenated data under coalescence. Systematic Biology, 56, 17-24.

Lamichhaney, S., Berglund, J., Almén, M. S., Maqbool, K., Grabherr, M., Martinez-Barrio, A., . . Zamani, N. (2015). Evolution of Darwin/'s finches and their beaks revealed by genome sequencing. Nature, 518, 371-375.

Larget, B. R., Kotha, S. K., Dewey, C. N., \& Ané, C. (2010). BUCKy: gene tree/species tree reconciliation with Bayesian concordance analysis. Bioinformatics, 26, 2910-2911.

Li, H., Handsaker, B., Wysoker, A., Fennell, T., Ruan, J., Homer, N., . . Durbin, R. (2009). The sequence alignment/map format and SAMtools. Bioinformatics, 25, 2078-2079.

Li, Y. F., Costello, J. C., Holloway, A. K., \& Hahn, M. W. (2008). "Reverse ecology" and the power of population genomics. Evolution, 62, 2984-2994.

Löytynoja, A., \& Goldman, N. (2005). An algorithm for progressive multiple alignment of sequences with insertions. Proceedings of the National Academy of Sciences of the United States of America, 102, 10557-10562.

Maddison, W. P. (1997). Gene trees in species trees. Systematic Biology, 46, 523-536.

Marin, E., Nussaume, L., Quesada, A., Gonneau, M., Sotta, B., Hugueney, P., . . Marion-Poll, A. (1996). Molecular identification of zeaxanthin epoxidase of Nicotiana plumbaginifolia, a gene involved in abscisic acid biosynthesis and corresponding to the ABA locus of Arabidopsis thaliana. The EMBO Journal, 15, 2331.

Martin, A., \& Orgogozo, V. (2013). The loci of repeated evolution: a catalog of genetic hotspots of phenotypic variation. Evolution, 67, 1235-1250.

Martin, S. H., Dasmahapatra, K. K., Nadeau, N. J., Salazar, C., Walters, J. R., Simpson, F., . . Jiggins, C. D. (2013). Genome-wide evidence for speciation with gene flow in Heliconius butterflies. Genome Research, 23, 1817-1828.

Mendes, F. K., Hahn, Y., \& Hahn, M. W. (2016). Gene tree discordance can generate patterns of diminishing convergence over time. Molecular Biology and Evolution, 33, 3299-3307.

Miller, R. J., Mione, T., Phan, H.-L., \& Olmstead, R. G. (2011). Color by numbers: Nuclear gene phylogeny of Jaltomata (Solanaceae), sister genus to Solanum, supports three clades differing in fruit color. Systematic Botany, 36, 153-162.

Mirarab, S., \& Warnow, T. (2015). ASTRAL-II: coalescent-based species tree estimation with many hundreds of taxa and thousands of genes. Bioinformatics, 31, i44-i52.

Moine, T. (1992). Systematics and evolution of Jaltomata (Solanaceae). Ph. D. Dissertation. Storrs, 
1001

1002

1003

1004

1005

1006

1007

1008

1009

1010

1011

1012

1013

1014

1015

1016

1017

Connecticut: University of Connecticut.

Mione T., S. Leiva González \& L. Yacher. (2015). Two new Peruvian species of Jaltomata (Solanaceae, Solaneae) with red floral nectar. Brittonia, 67, 105-112.

Novikova, P. Y., Hohmann, N., Nizhynska, V., Tsuchimatsu, T., Ali, J., Muir, G., . . . Fedorenko, O. M. (2016). Sequencing of the genus Arabidopsis identifies a complex history of nonbifurcating speciation and abundant trans-specific polymorphism. Nature Genetics, 48, 1077-1082.

Olmstead, R. G., Bohs, L., Migid, H. A., Santiago-Valentin, E., Garcia, V. F., \& Collier, S. M. (2008). A molecular phylogeny of the Solanaceae. Taxon, 57, 1159-1181.

Owens, G. L., Baute, G. J., \& Rieseberg, L. H. (2016). Revisiting a classic case of introgression: Hybridization and gene flow in Californian sunflowers. Molecular Ecology, 25, 2630-2643.

Pamilo, P., \& Nei, M. (1988). Relationships between gene trees and species trees. Molecular Biology and Evolution, 5, 568-583.

Paradis, E., Claude, J., \& Strimmer, K. (2004). APE: analyses of phylogenetics and evolution in R language. Bioinformatics, 20, 289-290.

Pease, J., \& Rosenzweig, B. (2015). Encoding data using biological principles: the Multisample Variant Format for phylogenomics and population genomics. IEEE/ACM Transactions on Computational Biology and Bioinformatics, doi:10.1109/tcbb.2015.2509997.

Pease, J. B., Haak, D. C., Hahn, M. W., \& Moyle, L. C. (2016). Phylogenomics reveals three sources of adaptive variation during a rapid radiation. PLoS Biology, 14, e1002379.

Pease, J. B., \& Hahn, M. W. (2015). Detection and polarization of introgression in a five-taxon phylogeny. Systematic Biology, 64, 651-662.

Ramsay, N. A., \& Glover, B. J. (2005). MYB-bHLH-WD40 protein complex and the evolution of cellular diversity. Trends in Plant Science, 10, 63-70.

Rausher, M. D. (2008). Evolutionary transitions in floral color. International Journal of Plant Sciences, 169, $7-21$.

Revell, L. J. (2012). phytools: an R package for phylogenetic comparative biology (and other things). Methods in Ecology and Evolution, 3, 217-223.

Richards, E. J., \& Martin, C. H. (2017). Adaptive introgression from distant Caribbean islands contributed to the diversification of a microendemic adaptive radiation of trophic specialist pupfishes. PLoS Genetics, 13, e1006919.

Salichos, L., \& Rokas, A. (2013). Inferring ancient divergences requires genes with strong phylogenetic signals. Nature, 497, 327-331.

Särkinen, T., Bohs, L., Olmstead, R. G., \& Knapp, S. (2013). A phylogenetic framework for evolutionary study of the nightshades (Solanaceae): a dated 1000-tip tree. BMC Evolutionary Biology, 13, 214.

Sela, I., Ashkenazy, H., Katoh, K., \& Pupko, T. (2015). GUIDANCE2: accurate detection of unreliable alignment regions accounting for the uncertainty of multiple parameters. Nucleic Acids Research, 43, W7-W14.

Simkin, A. J., Schwartz, S. H., Auldridge, M., Taylor, M. G., \& Klee, H. J. (2004). The tomato carotenoid cleavage dioxygenase 1 genes contribute to the formation of the flavor volatiles $\beta$ - ionone, pseudoionone, and geranylacetone. The Plant Journal, 40, 882-892.

Specht, C. D., \& Howarth, D. G. (2015). Adaptation in flower form: a comparative evodevo approach. New Phytologist, 206, 74-90.

Stamatakis, A. (2006). RAxML-VI-HPC: maximum likelihood-based phylogenetic analyses with thousands of taxa and mixed models. Bioinformatics, 22, 2688-2690.

Stern, D. L. (2013). The genetic causes of convergent evolution. Nature Reviews Genetics, 14, 751-764.

Storz, J. F. (2016). Causes of molecular convergence and parallelism in protein evolution. Nature Reviews Genetics, 17, 239-250.

The Tomato Genome Consortium. (2012). The tomato genome sequence provides insights into fleshy fruit evolution. Nature, 485, 635-641.

Venkat, A., Hahn, M. W., \& Thornton, J. W. (2017). Multinucleotide mutations cause false inferences of positive selection. bioRxiv. doi:10.1101/165969

Wake, D. B., Wake, M. H., \& Specht, C. D. (2011). Homoplasy: from detecting pattern to determining process and mechanism of evolution. Science, 331, 1032-1035.

Wickett, N. J., Mirarab, S., Nguyen, N., Warnow, T., Carpenter, E., Matasci, N., . . Gitzendanner, M. A. (2014). Phylotranscriptomic analysis of the origin and early diversification of land plants. Proceedings of the National Academy of Sciences of the United States of America, 111, E4859- 
1018

1019

1020

1021

1022

1023

1024

1025

1026

1027

1028

1029

1030

1031

1032

1033

\section{E4868.}

Wuest, S. E., O’Maoileidigh, D. S., Rae, L., Kwasniewska, K., Raganelli, A., Hanczaryk, K., . . Wellmer, F. (2012). Molecular basis for the specification of floral organs by APETALA3 and PISTILLATA. Proceedings of the National Academy of Sciences, 109, 13452-13457.

Yang, Y., Moore, M. J., Brockington, S. F., Soltis, D. E., Wong, G. K.-S., Carpenter, E. J., . . Xie, Y. (2015). Dissecting molecular evolution in the highly diverse plant clade Caryophyllales using transcriptome sequencing. Molecular Biology and Evolution, 32, 2001-2014.

Yang, Y., \& Smith, S. A. (2014). Orthology inference in nonmodel organisms using transcriptomes and low-coverage genomes: improving accuracy and matrix occupancy for phylogenomics. Molecular Biology and Evolution, 31, 3081-3092.

Yang, Z. (2007). PAML 4: phylogenetic analysis by maximum likelihood. Molecular Biology and Evolution, 24, 1586-1591.

Yuan, H., Zhang, J., Nageswaran, D., \& Li, L. (2015). Carotenoid metabolism and regulation in horticultural crops. Horticulture Research, 2, 15036. 


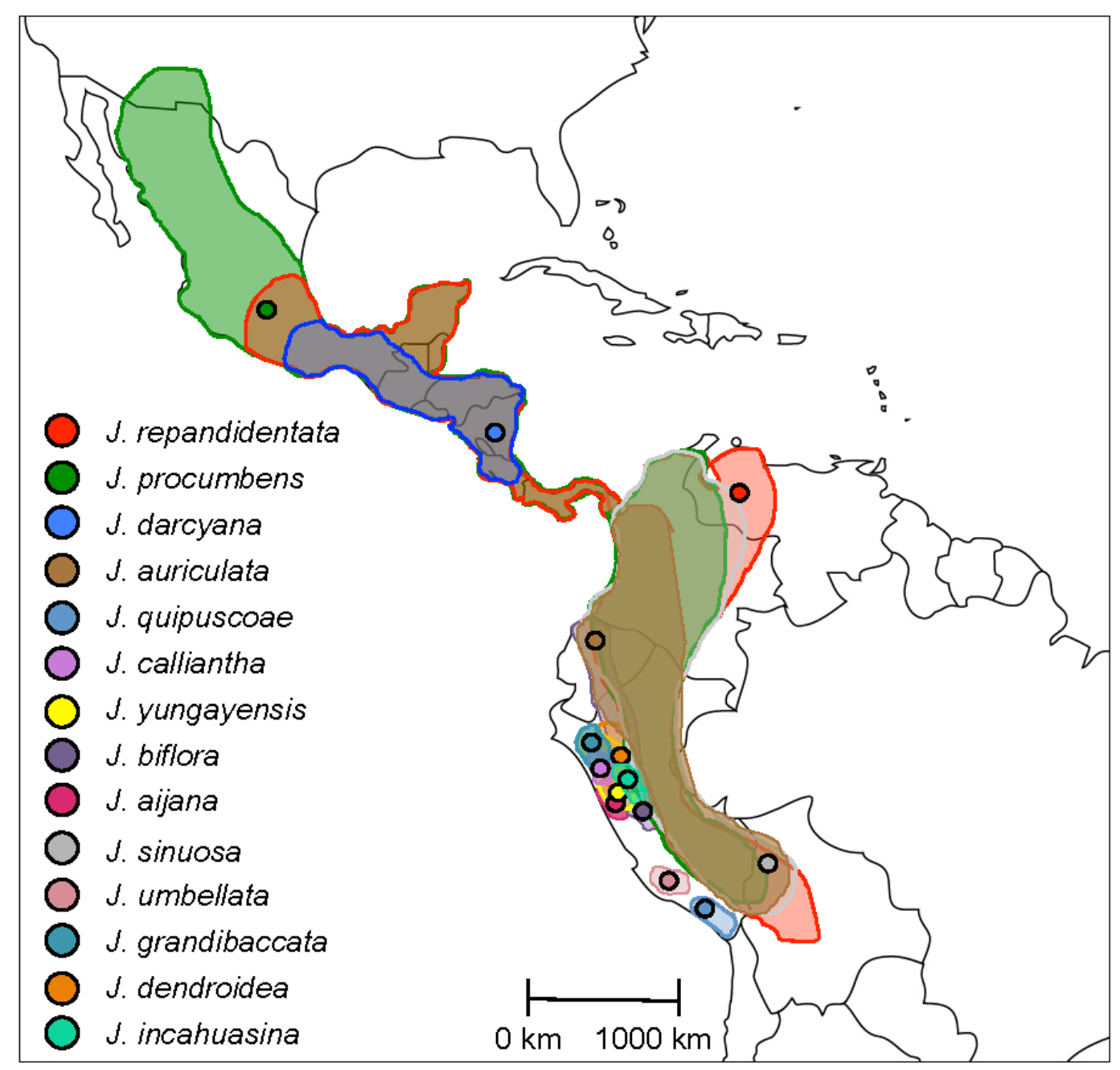

Fig 1. Geographic distribution of investigated Jaltomata species. For each species, the sample location is labeled. Ranges estimated from herbarium specimens (T. Mione and S. Leiva G., pers. comm.; J. L. Kostyun, unpub.). 
bioRxiv preprint doi: https://doi.org/10.1101/201376; this version posted October 11,2017 . The copyright holder for this preprint (which was not certified by peer review) is the author/funder, who has granted bioRxiv a license to display the preprint in perpetuity. It is made available under

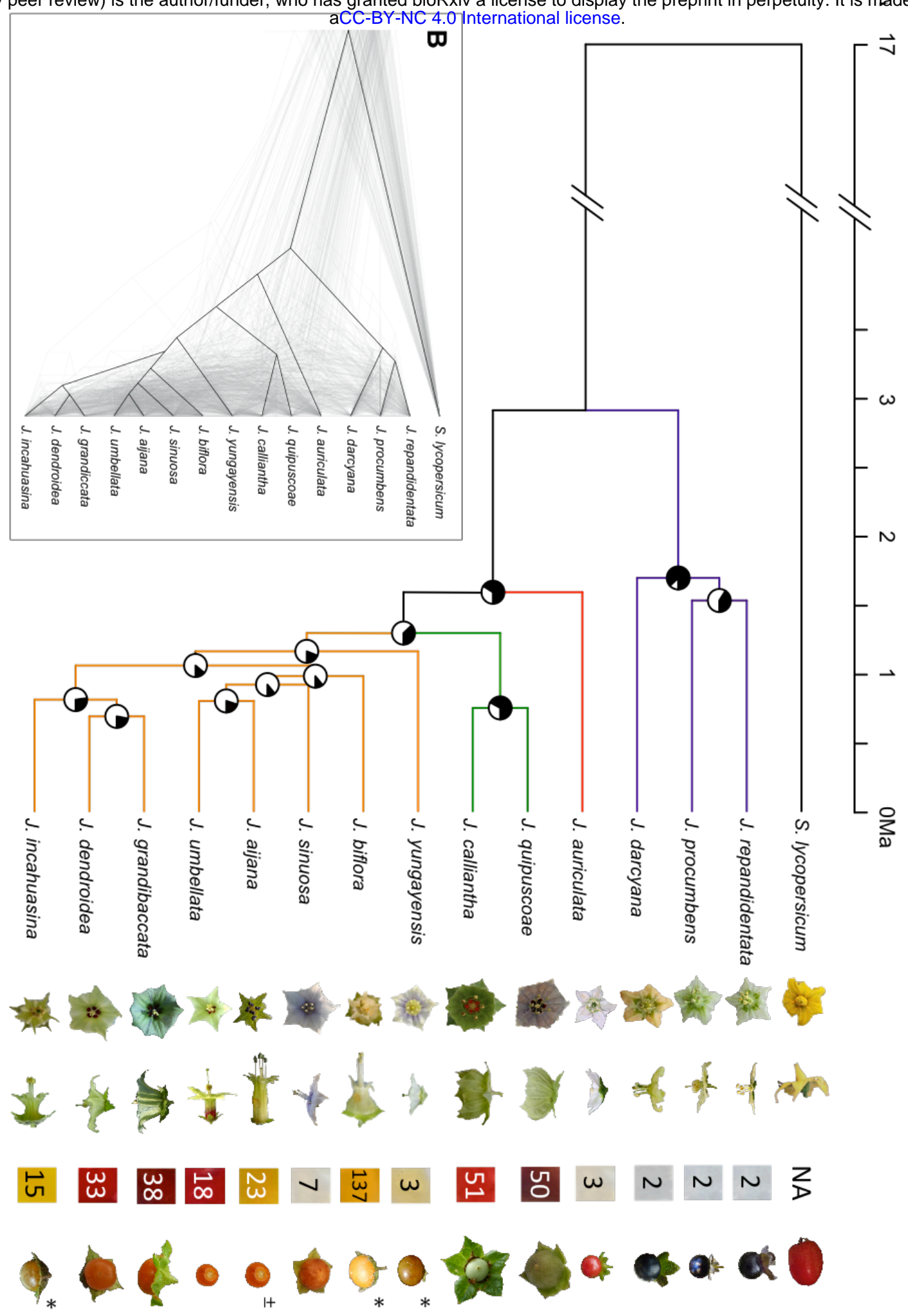

Fig 2. The phylogeny of investigated Jaltomata species. (A) A whole-transcriptome concatenated phylogeny of Jaltomata species with Solanum lycopersicum as outgroup. The Pie chart on each internode shows the concordance factor estimated from BUCKy, with the amount of black representing the degree of concordance. Divergence times estimated in ape with 17MYA Jaltomata-Solanum calibration from (Särkinen et al., 2013). Representative flower and fruit images to the right of species names: front view of flower, lateral view of flower, nectar color and volume $(\mathrm{uL})$ per flower, and ripe fruit. Image with \pm indicates that fruit from a similar species is shown, and * indicates contributed by Dr. Thomas Mione at Central Connecticut State University. 
bioRxiv preprint doi: https://doi.org/10.1101/201376; this version posted October 11,2017 . The copyright holder for this preprint (which was not certified by peer review) is the author/funder, who has granted bioRxiv a license to display the preprint in perpetuity. It is made available under aCC-BY-NC 4.0 International license.

(B) A 'cloudogram' of 183 gene trees whose average bootstrap values are larger than 70 across the nodes. For contrast, the concatenated tree (Fig 2A) is shown in black. 

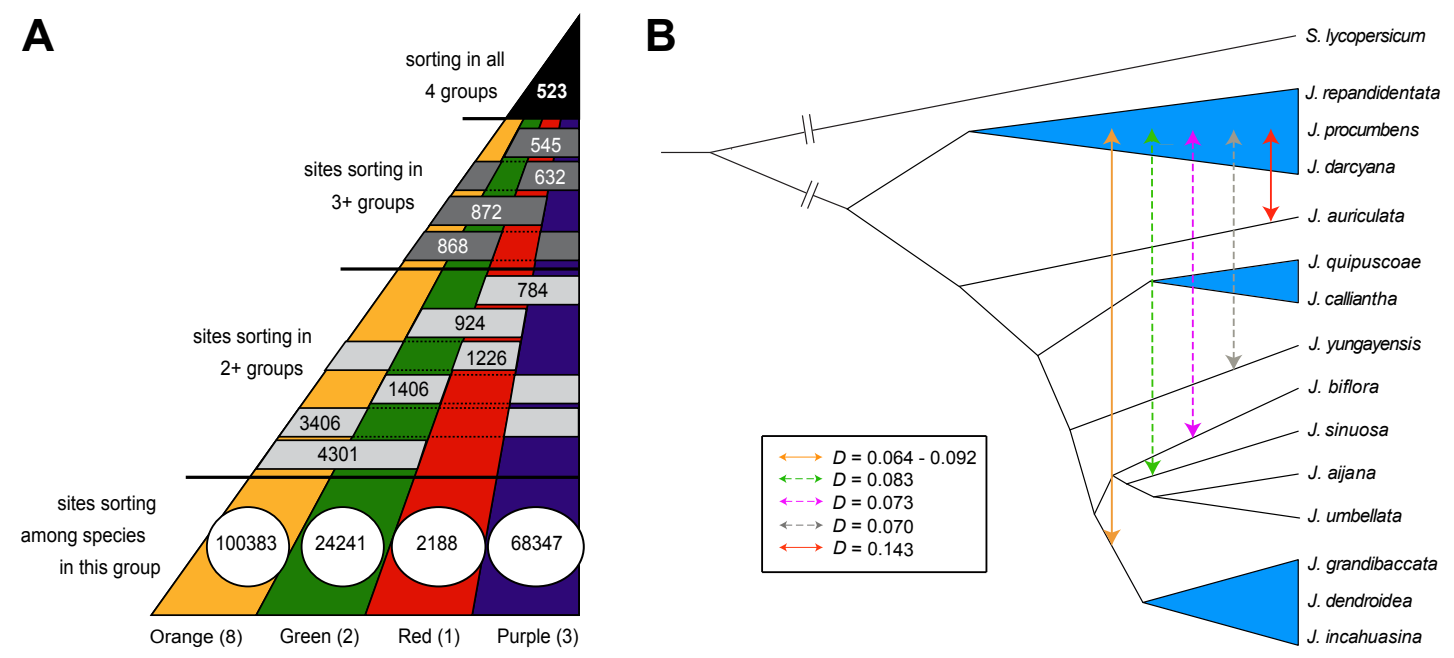

Fig 3. (A) Allele sorting, with the number of genetic variants within or shared between each Jaltomata subclade. (B) The introgression pattern among Jaltomata lineages. The solid lines indicate strong evidence of introgression between two lineages or sub-clades, while the dashed lines indicate putative introgression. The corresponding Patterson's $D$ statistic value is labeled for each putative introgression event. 
bioRxiv preprint doi: https://doi.org/10.1101/201376; this version posted October 11,2017 . The copyright holder for this preprint (which was not certified by peer review) is the author/funder, who has granted bioRxiv a license to display the preprint in perpetuity. It is made available under aCC-BY-NC 4.0 International license.
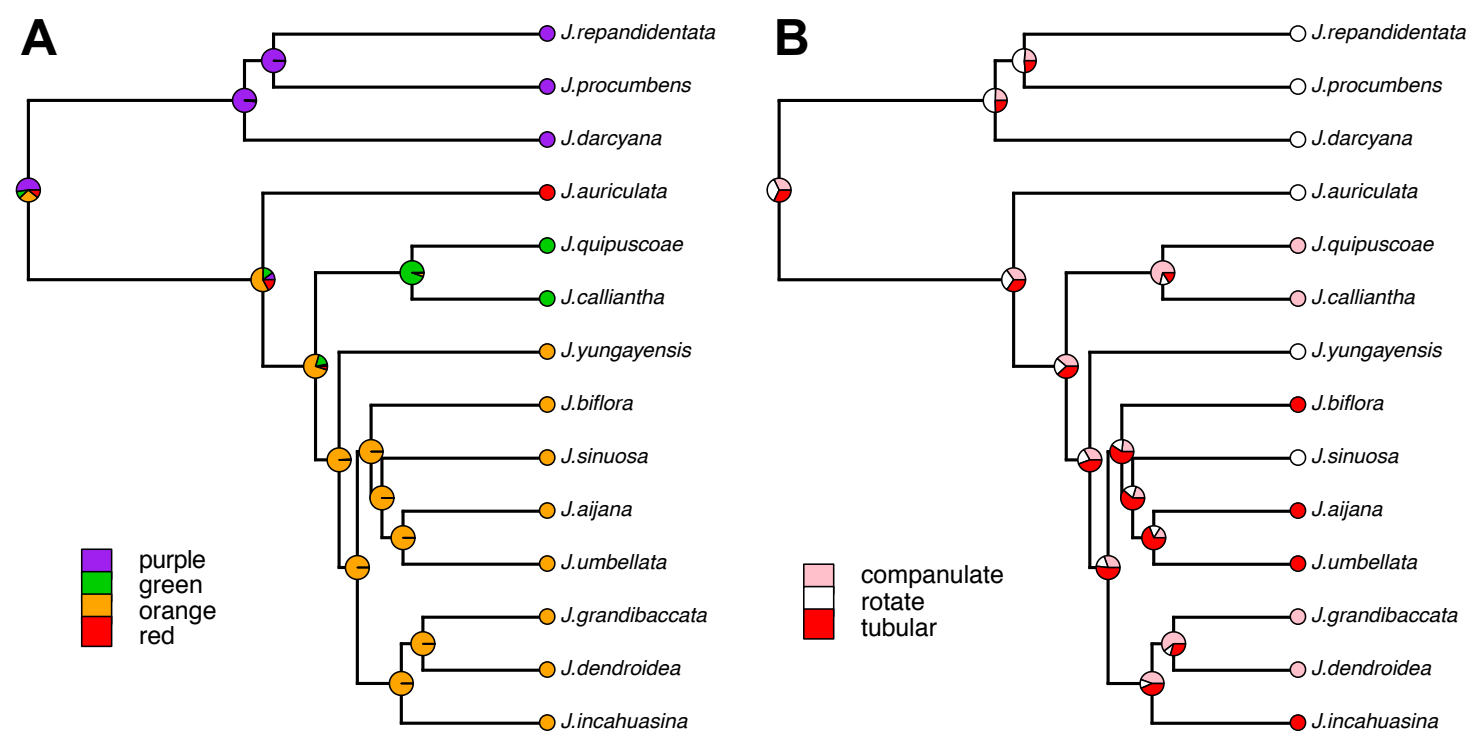

Fig 4. Ancestral character-state reconstruction of (A) fruit color, (B) corolla shape in investigated Jaltomata using maximum likelihood. 


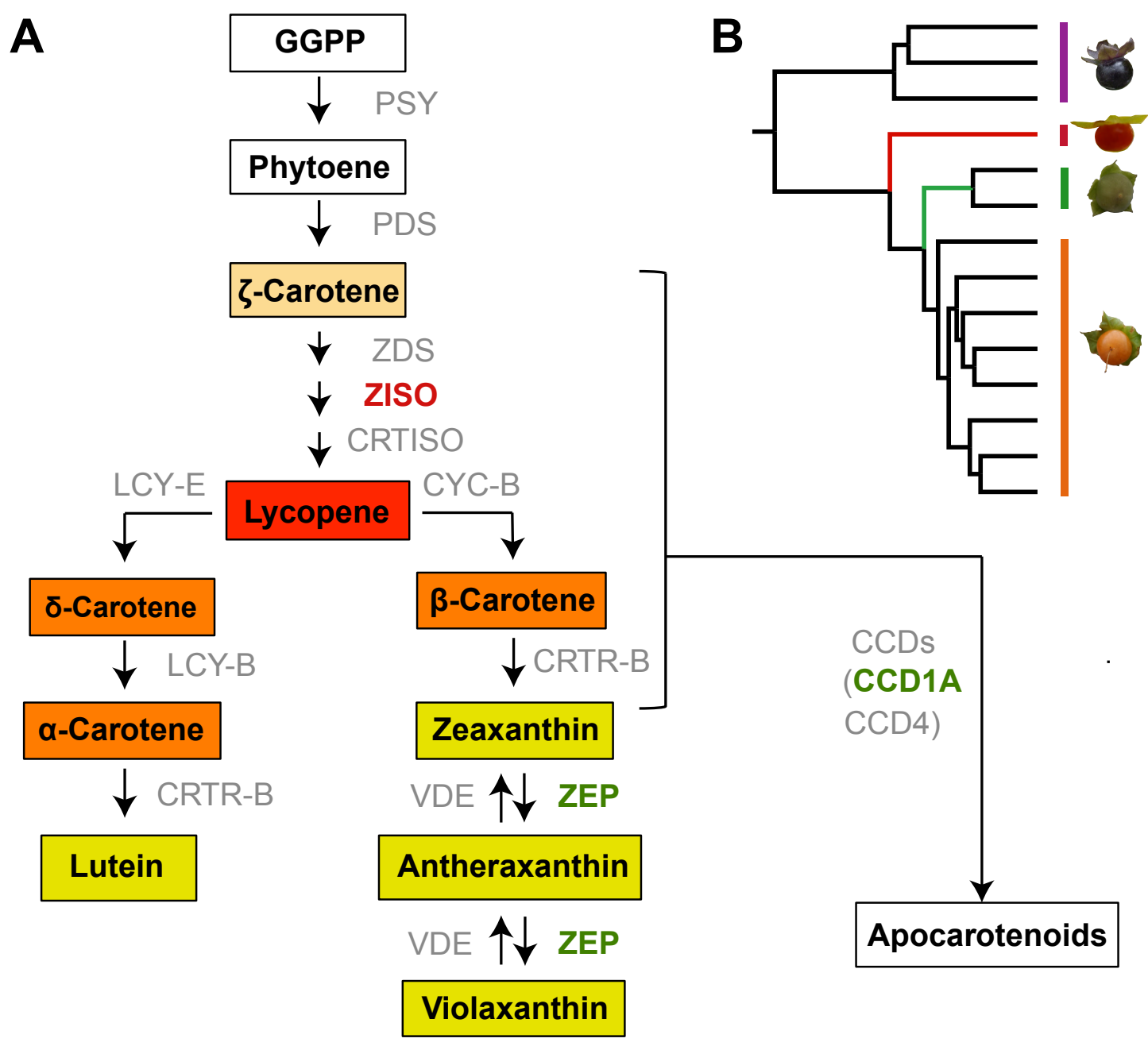

Fig 5. Genes under adaptive evolution in the carotenoid biosynthesis pathway. (A) Simplified carotenoid biosynthesis pathway modified from (Yuan et al. 2015). Genes under adaptive evolution are indicated by their names highlighted in colors corresponding to particular branches (see panel B). $P S Y$, phytoene synthase; $P D S$, phytoene desaturase; $Z D S$, $\zeta$-carotene desaturase; ZISO, $\zeta$-carotene isomerase; $C R T I S O$, carotenoid isomerase; $L C Y$-E, lycopene $\varepsilon$-cyclase; $L Y C$ - $B$, lycopene $\beta$-cyclase; $C R T R$ - $B, \beta$-ring hydroxylase; $C Y C$ - $B$, chromoplast specific lycopene $\beta$ cyclase; $Z E P$, zeaxanthin epoxidase; $V D E$, violaxanthin de-epoxidase; $C C D$, carotenoid cleavage dioxygenase. Metabolites are boxed and colored according to their compound colors, whereas white boxes indicate no color. (B) Positive selection signatures of genes on different branches are indicated by different colors: red-fruited lineages (Red), and green-fruited lineages (Green). 